\title{
Is There a Future for 74 Gy Radiation Treatment of NSCLC after RTOG 0617? A Comparison of the RTOG Study Results with Our Own Department's 74 Gy NSCLC Cohort
}

\author{
Christina Schröder ${ }^{1,2 *}$, Eyck Blank ${ }^{1}$, Dietrich Sidow ${ }^{1}$, Rita Engenhart-Cabillic ${ }^{2}$, \\ André Buchali ${ }^{1}$ \\ ${ }^{1}$ Departement of Radiooncology, Ruppiner Kliniken GmbH, Neuruppin, Germany \\ ${ }^{2}$ Departement of Radiooncology, University Hospital, Justus Liebig University, Giessen and Marburg, Germany \\ Email: ${ }^{*}$ christina.schroeder@med.uni-giessen.de
}

Received 18 May 2015; accepted 25 July 2015; published 28 July 2015

Copyright (C) 2015 by authors and Scientific Research Publishing Inc. This work is licensed under the Creative Commons Attribution International License (CC BY). http://creativecommons.org/licenses/by/4.0/ (c) (i) Open Access

\begin{abstract}
Background and Purpose: There have been a number of different efforts trying to improve the outcome of NSCLC patients treated with radiotherapy (RT). Contrary to most expectations, the long awaiting results of the RTOG 0617 trial didn't show any benefit of dose escalation to $74 \mathrm{~Gy}$. In this unicentric retrospective analysis we compare the RTOG 0617 result with the outcome of our own 74-Gy-NSCLC cohort. Methods and Material: Since October 2009, 80 patients with NSCLC were treated with $74 \mathrm{~Gy}$ in 37 fractions, of which 69 patients were eligible for a retrospective analysis of local and distant failure, survival time and treatment related toxicity. A subgroup analysis was done for patients with a possible follow-up of at least 18 month. Results: Complete local remission could be achieved in 18 patients $(26.1 \%) ; 26$ patients $(37.7 \%)$ had a partial remission and 3 patients (4.4\%) a stable local disease. Local failure occurred in 12 patients (17.3\%). Distant failure occurred in 27 patients (39.1\%). The median survival time was 43.7 weeks (95\% CI: 25.2 - 62.3 weeks). 5 patients $(6.3 \%)$ developed RT induced side effects. As for the analyzed subgroup, a complete or partial local remission could be achieved in 29 patients $(61.7 \%)$. Local failure occurred in 11 patients (23.4\%) and 20 patients (42.6\%) developed distant metastases. The 18-month overall survival was $38.3 \%$ and the median survival time was 51.7 weeks (95\% CI: $27.2-76.3$ weeks). Conclusion: The results of this retrospective analysis indicate that $74 \mathrm{~Gy}$ total radiation dose might not lead to results as bad as indicated by the RTOG 0617 trial. It might therefore be a suitable treatment concept for people with NSCLC.
\end{abstract}

\footnotetext{
${ }^{*}$ Corresponding author.
}

How to cite this paper: Schröder, C., Blank, E., Sidow, D., Engenhart-Cabillic, R. and Buchali, A. (2015) Is There a Future for 74 Gy Radiation Treatment of NSCLC after RTOG 0617? A Comparison of the RTOG Study Results with Our Own Department's 74 Gy NSCLC Cohort. Journal of Cancer Therapy, 6, 642-647. http://dx.doi.org/10.4236/jct.2015.68071 


\section{Keywords}

\section{Lung Cancer, Dose Escalation, Local Remission, Survival, Side Effects}

\section{Introduction}

The outcome of lung cancer patients treated with radiotherapy (RT) is still poor. The 5-year overall survival is reported to be somewhere between $8 \%$ and $21 \%$. For stage IIIb+ disease these values rapidly decrease to $5 \%$ or less [1].

There have been a number of different efforts trying to improve the outcome. One apparent option seemed to be dose escalation, especially since new technologies like IMRT or VMAT made it possible to deliver a higher treatment dose with better conformity of the target volume and a better sparing of organs at risk [2] [3].

Contrary to most expectations, the long awaiting results of the RTOG 0617 trial didn't show any benefit of dose escalation and opened a variety of questions [4]. Why would a higher treatment dose lead to a worse outcome, especially since RT induced adverse events (AE) don't seem to be the problem? Since the publications of the results numerous efforts have been made trying to explain the unexpected results of the trial, however common consent seems to be missing [5].

In this unicentric retrospective analysis we compare the RTOG 0617 result with the outcome of our own 74Gy-NSCLC cohort. Similar to the trial, we also focused on the endpoints local and distant control, survival and RT induced toxicity.

\section{Material and Methods}

With regards to treatment related side effects and the introduction of intensity modulated and volumetric arc irradiation techniques the total radiation dose in our department was raised stepwise from 66 Gy to 70 Gy to 74 Gy between May 2004 and October 2009. All patients signed an informed consent.

Since then, 80 patients with non small cell lung cancer (NSCLC) were treated with 74 Gy in 37 fractions with or without concurrent or sequential chemotherapy. The histology types and tumor stages are shown in Table 1. 11 patients with partial or complete lobectomy in their medical history were excluded from analysis. 69 patients were eligible for a retrospective analysis of local and distant failure, survival time and treatment related toxicity.

The mean age of patients was 67 years (51 - 82 years) at the time of therapy. Most patients (72.5\%) had stage IIIb disease or higher.

41 (59.4\%) patients received concurrent chemotherapy (cisplatinum $80 \mathrm{mg} / \mathrm{m}^{2}$, d1 and Vinorelbine $15 \mathrm{mg} / \mathrm{m}^{2}$, d1.8) every 3 weeks with a maximum of 4 cycles, 32 of the patients received 2 or more cycles. 10 patients (14.5\%) received sequential chemotherapy and 18 (26.1\%) patients were unable to receive chemotherapy due to reduced performance status and comorbidities.

Further patient characteristics are shown in Table 1.

Patients were treated using either intensity modulated radiotherapy (IMRT) with "sliding windows"

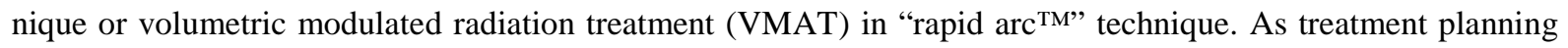
system Eclipse ${ }^{\mathrm{TM}}$ (Varian Medical Systems) with a pencil beam and since June 2011 an AAA-algorithm was used. As for contouring, the macroscopic tumor plus the macroscopically involved lymph nodes (dose painting) were included in the gross tumor volume (GTV) receiving a total treatment dose of $74 \mathrm{~Gy}$. The ipsilateral hilar lymphatic drainage and if macroscopically involved also the ipsilateral mediastinal and contralateral hilar lymphatic drainage received a treatment dose of $60 \mathrm{~Gy}$ in one simultaneous integrated boost plan.

Dose constrains for treatment planning were an EQD2-equivalent $V_{20 G y}$ of $<30 \%$ and a $V_{30 G y}$ of $<20 \%$ for the entire lung with an estimated $\alpha / \beta$ value of 2 . As for the esophagus, the maximum dose $\left(\mathrm{D}_{\max }\right)$ was $74 \mathrm{~Gy}$. Patients received a clinical restaging 3 months after therapy. Either this restaging data, or if available, data from later appointments was used for analysis. Due to the possibility of a bias because of the short follow up time in some patients a subgroup analysis was done with patients with a possible follow up $>18$ months. For statistical analysis SPSS version 22 was used. 
Table 1. Patient characteristics.

\begin{tabular}{|c|c|c|}
\hline & No. of patients & $(\%)$ \\
\hline Total & 69 & 100.0 \\
\hline \multicolumn{3}{|l|}{ Tumor stage } \\
\hline IIa & 3 & 4.3 \\
\hline $\mathrm{IIb}$ & 2 & 2.9 \\
\hline IIIa & 14 & 20.3 \\
\hline IIIb & 50 & 72.5 \\
\hline \multicolumn{3}{|l|}{ Histology } \\
\hline Adenocarcinoma & 23 & 33.3 \\
\hline Squamous & 40 & 58.0 \\
\hline Others & 5 & 7.3 \\
\hline None & 1 & 1.4 \\
\hline \multicolumn{3}{|l|}{ Sex } \\
\hline Male & 58 & 84.1 \\
\hline Female & 11 & 15.9 \\
\hline \multicolumn{3}{|l|}{ Chemotherapy } \\
\hline None & 18 & 26.1 \\
\hline Sequential & 9 & 13.0 \\
\hline Concurrent & 35 & 50.7 \\
\hline Concurrent + sequential & 6 & 8.8 \\
\hline Unknown & 1 & 1.4 \\
\hline \multicolumn{3}{|l|}{ Age } \\
\hline $45-54$ & 6 & 8.8 \\
\hline $55-64$ & 19 & 27.5 \\
\hline $65-74$ & 28 & 40.6 \\
\hline$\geq 75$ & 16 & 23.1 \\
\hline Mean & 67 & \\
\hline SD & 9 & \\
\hline
\end{tabular}

\section{Results}

The median follow up of all patients was 33 weeks (range 1 - 188 weeks). At time of analysis 21 patients were still alive.

\subsection{Local Control}

Complete local remission could be achieved in 18 patients (26.1\%), 26 patients (37.7\%) had a partial remission and 3 patients (4.3\%) a stable local disease. Data regarding local control were missing in 10 patients (14.4\%), which either died shortly after RT $(n=8)$ or follow-up was otherwise lost $(n=2)$. Local failure within the target volume occurred in 10 patients $(14.4 \%)$ and two patients $(2.9 \%)$ had a local recurrence outside the contoured target volume. 


\subsection{Distant Control}

Distant failure occurred in 27 patients (39.1\%). 19 patients with distant failure were treated with concurrent or sequential chemotherapy. Follow up data regarding distant control were missing in 4 patients (5.8\%).

\subsection{Survival}

At the time of analysis 21 patients (30.4\%) were still alive. Survival data of 4 patients (5.8\%) were missing. The median survival time was 43.7 weeks (95\% CI: 25.2 - 62.3 weeks). The most common cause of death was the underlying oncological disease $(n=34)$, followed by cardiac causes $(n=4)$ and non-tumor related acute events $(n=4)$. One patient died of radiation induced pneumonia and cause of death is missing in one patient.

The Kaplan Meier curve of patients is shown in Figure 1.

\subsection{Toxicity and Dose Values}

Only two patients (2.9\%) developed grade 3 dysphagia after treatment. Three patients (3.4\%) developed RT induced pneumonia grade III by 3 month after treatment, which was still present at the 6 month follow up in two patients (2.9\%). One patient died of RT induced pneumonia.

The numeric dose values were a mean lung dose of $19.5 \mathrm{~Gy}$ (standard deviation $7.5 \mathrm{~Gy}$ ), a $\mathrm{V}_{20 \mathrm{~Gy}}$ of $35.4 \%$ (SD 9.3\%) and a $\mathrm{V}_{30 \mathrm{~Gy}}$ of 22.1\% (SD 4.8\%). The EQD2 equivalent $\mathrm{V}_{20 \mathrm{~Gy}}$ and $\mathrm{V}_{30 \mathrm{~Gy}}$ were $23.9 \%$ and $14.6 \%$, respectively. As for the esophagus, the mean $\mathrm{D}_{\max }$ was $73 \mathrm{~Gy}$ (SD $2.4 \mathrm{~Gy}$ ), $\mathrm{V}_{60 \mathrm{~Gy}}$ was $40.9 \%$ (SD 20.1\%) and $\mathrm{V}_{70 \mathrm{~Gy}}$ was $10.6 \%$ (SD 9.3\%).

\subsection{Subgroup Analysis (Patients with Follow up > 18 Month)}

47 patients with NSCLC were treated until 08/13. A complete local remission could be achieved in 15 patients (31.9\%) and 14 patients (29.8\%) had a partial remission in their latest follow-up CT. Data regarding local control were missing in 7 patients (14.9\%), which died shortly after treatment. Local failure within the target volume occurred in 10 patients (21.3\%) and one patient $(2.1 \%)$ had a recurrence outside the target volume. As for distant failure, 20 (42.6\%) patients developed metastases. Data of 3 patients (6.4\%) was missing. The 18-month overall survival was $38.3 \%$ and the median survival time was 51.7 weeks (95\% CI 27.2 - 76.3 weeks).

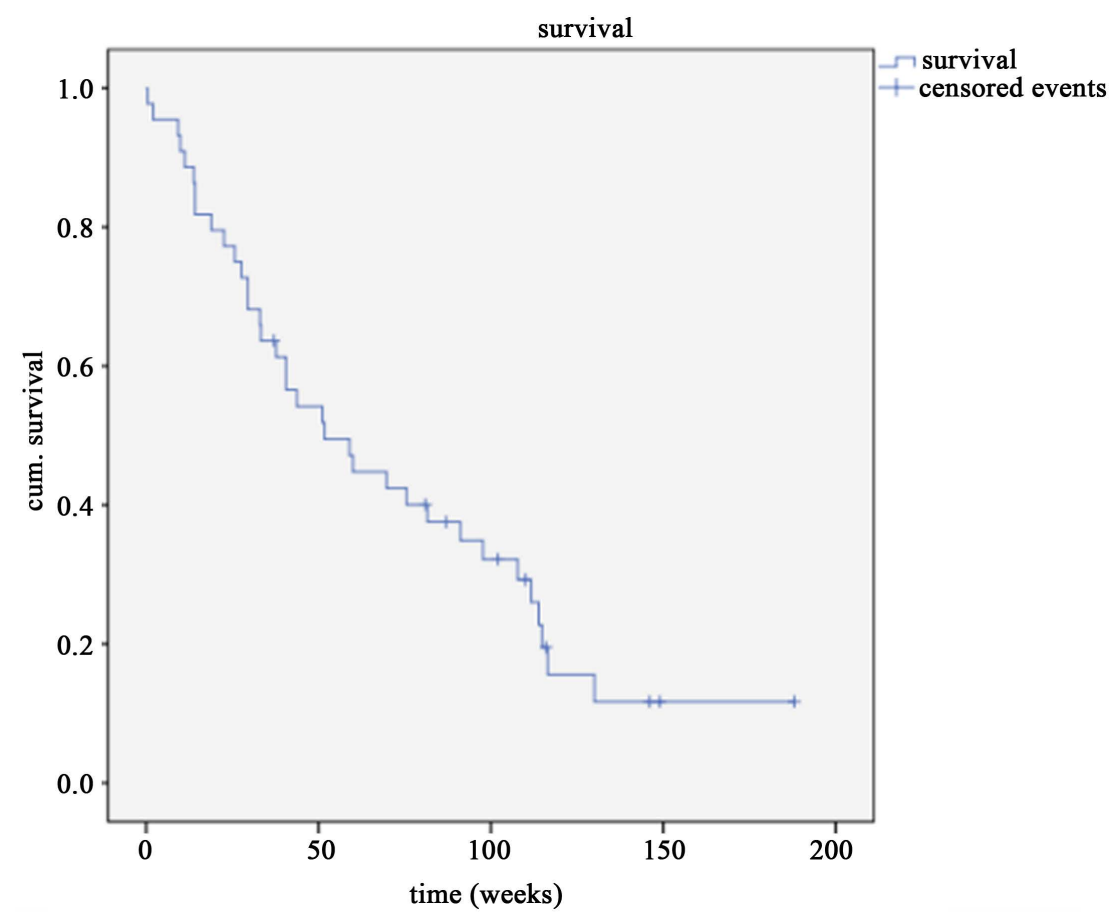

Figure 1. Survival of patients. 


\section{Discussion}

Compared to the RTOG 0617 results in their HD 74 Gy arm we achieved better results for local failure (17.3\% vs. $34.3 \%$ RTOG), both the grade $3+$ and the grade 5 AEs (6.3 vs. $78.2 \%$ RTOG and 1 vs. 10 patients) and better results for distant failure (39.1\% vs. $47.8 \%$ RTOG). Regarding local and distant failure, our results were even better than the RTOG SD 60 Gy arm (25.1\% local and 42.2\% distant). However, the median survival time was worse (43.7 weeks vs. 19.5 month RTOG).

If focusing on the subgroup treated until $02 / 13$, the data concerning local and distant control were still better than those of RTOG 0617. The 18 month OS was $38.3 \%$, which was worse than the $53.9 \%$ of the RTOG. So why would OS be worse, if both local and distant control are better? One possible explanation might be patient selection. Only 35 patients (50.7\%) of the 69 treated in our department where matching the RTOG 0617 criteria (see ClinicalTrials.gov identifier NCT00533949). Common RTOG exclusion criteria which can be found in our cohort are no concurrent chemotherapy (26.1\%) and no stage IIIa/IIIb disease (7.2\%). The topic of poor OS after therapy rather opens up the question whether patients should be selected more carefully for such an aggressive curative treatment. For some of these patients palliative treatment might have been a good alternative [6].

Another aspect, the significantly lower rate of severe side effects in our cohort as compared to the RTOG cohort, might be explained with the use of modern RT delivery techniques like IMRT or VMAT, which made it possible to achieve a good sparing of OAR without having to compromise the treatment volume [2] [3]. In a number of different trials a total treatment dose of 74 Gy and higher proved to be safely deliverable with only a few severe side effects [7]-[12]. Unfortunately, data regarding the advantage or disadvantage of 74 Gy treatment compared to other treatment schedules with or without concurrent chemotherapy are rare and inconclusive [13] [14]. Usually, these analysis include patients with lower tumor stages and better performance status than we treated in our cohort, possibly due to the fact that in Germany, as compared e.g. to the U.S., patients are more likely to get lung surgery, especially with a stage IIIa disease or lower.

Besides dose escalation, numerous efforts have been made to improve survival by using alternative dose concepts like hyper-or hypofractionation. Again, the results regarding the benefit of either technique seem to be inconclusive [15]-[19]. Furthermore, the use of modern technologies beyond IMRT to optimize radiation treatment might open up interesting possibilities that might help to improve the still poor outcome of NSCLC patients [20].

\section{Conclusion}

The results of this retrospective analysis indicate that 74 Gy total radiation dose might not lead to results as bad as indicated by the RTOG 0617 trial. It might therefore be a suitable treatment concept for people with NSCLC.

\section{Conflict of Interest}

On behalf of all authors, the corresponding author states that there is no conflict of interest.

\section{Compliance with Ethical Guidelines}

The accompanying manuscript does not include studies on humans or animals.

\section{References}

[1] Bradley, J.D., Moughan, J., Graham, M.V., et al. (2010) A Phase I/II Radiation Dose Escalation Study with Concurrent Chemotherapy for Patients with Inoperable Stages I to III Non-Small-Cell Lung Cancer: Phase I Results of RTOG 0117. International Journal of Radiation Oncology, Biology, Physics, 77, 367-372. http://dx.doi.org/10.1016/j.ijrobp.2009.04.029

[2] Bradley, J. (2005) A Review of Radiation Dose Escalation Trials for Non-Small Cell Lung Cancer within the Radiation Therapy Oncology Group. Seminars in Oncology, 32, 111-113. http://dx.doi.org/10.1053/j.seminoncol.2005.03.020

[3] Bradley, J.D., Paulus, R., Komaki, R., et al. (2013) A Randomized Phase III Comparison of Standard-Dose (60 Gy) versus High-Dose (74 Gy) Conformal Chemoradiotherapy with or without Cetuximab for Stage III Non-Small Cell Lung Cancer: Results on Radiation Dose in RTOG 0617. ASCO Meeting Abstracts, 31, 7501.

[4] Chi, A., Nguyen, N.P., Welsh, J.S., et al. (2014) Strategies of Dose Escalation in the Treatment of Locally Advanced Non-Small Cell Lung Cancer: Image Guidance and beyond. Frontiers in Oncology, 4, 156. 
http://dx.doi.org/10.3389/fonc.2014.00156

[5] Cox, J.D. (2012) Are the Results of RTOG 0617 Mysterious? International Journal of Radiation Oncology, Biology, Physics, 82, 1042-1044. http://dx.doi.org/10.1016/j.ijrobp.2011.12.032

[6] Guckenberger, P.D.M., Kavanagh, A. and Partridge, M. (2012) Combining Advanced Radiotherapy Technologies to Maximize Safety and Tumor Control Probability in Stage III Non-Small Cell Lung Cancer. Strahlentherapie und Onkologie, 188, 894-900. http://dx.doi.org/10.1007/s00066-012-0161-9

[7] Harada, H., Nishio, M., Murakami, H., et al. (2013) Dose-Escalation Study of Three-Dimensional Conformal Thoracic Radiotherapy with Concurrent S-1 and Cisplatin for Inoperable Stage III Non-Small-Cell Lung Cancer. Clinical Lung Cancer, 14, 440-445. http://dx.doi.org/10.1016/j.cllc.2013.01.003

[8] Howlader, N., Noone, A.M., Krapcho, M., et al. (2014) SEER Cancer Statistics Review, 1975-2011. National Cancer Institute, Bethesda. http://seer.cancer.gov/csr/1975_2011/

[9] Kong, F.M., Hayman, J.A. and Griffith, K.A. (2006) Final Toxicity Results of a Radiation-Dose Escalation Study in Patients with Non-Small-Cell Lung Cancer (NSCLC): Predictors for Radiation Pneumonitis and Fibrosis. International Journal of Radiation Oncology, Biology, Physics, 65, 1075-1086. http://dx.doi.org/10.1016/j.jjrobp.2006.01.051

[10] Liao, Z.X., Komaki, R.R., Thames Jr., H.D., et al. (2010) Influence of Technologic Advances on Outcomes in Patients with Unresectable, Locally Advanced Non-Small-Cell Lung Cancer Receiving Concomitant Chemoradiotherapy. International Journal of Radiation Oncology, Biology, Physics, 76, 775-781. http://dx.doi.org/10.1016/j.ijrobp.2009.02.032

[11] Partridge, M., Ramos, M., Sardaro, A. and Brada, M. (2011) Dose Escalation for Non-Small Cell Lung Cancer: Analysis and Modelling of Published Literature. Radiotherapy and Oncology, 99, 6-11. http://dx.doi.org/10.1016/j.radonc.2011.02.014

[12] Philipp, J. and Baumann, M. (2014) Caution with Hypofractionated Dose-Escalating Radiotherapy in Non-Small Cell Lung Cancer. Strahlentherapie und Onkologie, 190, 597-599. http://dx.doi.org/10.1007/s00066-014-0667-4

[13] Rosenman, J.G., Halle, J.S., Socinski, M.A., et al. (2002) High-Dose Conformal Radiotherapy for Treatment of Stage IIIA/IIIB Non-Small-Cell Lung Cancer: Technical Issues and Results of a Phase I/II Trial. International Journal of Radiation Oncology, Biology, Physics, 54, 348-356. http://dx.doi.org/10.1016/S0360-3016(02)02958-9

[14] Saunders, M., Royas, A., Lyn, E., et al. (1998) Experience with Dose Escalation Using CHARTWEL (Continuous Hyperfractionated Accelerated Radiotherapy Weekend Less) in Non-Small-Cell Lung Cancer. British Journal of Cancer, 78, 1323-1328. http://dx.doi.org/10.1038/bjc.1998.678

[15] Saunders, M., Dische, S., Barrett, A., et al. (1997) Continuous Hyperfractionated Accelerated Radiotherapy (CHART) versus Conventional Radiotherapy in Non-Small-Cell Lung Cancer: A Randomised Multicentre Trial. The Lancet, 350, 161-165. http://dx.doi.org/10.1016/S0140-6736(97)06305-8

[16] Schröder, C., Ivo, M. and Buchali, A. (2013) Does High-Dose Radiotherapy Benefit Palliative Lung Cancer Patients? Strahlentherapie und Onkologie, 189, 771-776. http://dx.doi.org/10.1007/s00066-013-0360-z

[17] Socinski, M.A., Morris, D.E. and Halle, J.S. (2004) Induction and Concurrent Chemotherapy with High-Dose Thoracic Conformal Radiation Therapy in Unresectable Stage IIIA and IIIB Non-Small-Cell Lung Cancer: A Dose-Escalation Phase I Trial. Journal of Clinical Oncology, 22, 4341-4350. http://dx.doi.org/10.1200/JCO.2004.03.022

[18] Terakedis, B. and Sause, W. (2011) Radiation Dose Escalation in Stage III Non-Small-Cell Lung Cancer. Frontiers in Oncology, 1, 47. http://dx.doi.org/10.3389/fonc.2011.00047

[19] Wang, L., Correa, C.R., Zhao, L., et al. (2009) The Effect of Radiation Dose and Chemotherapy on Overall Survival in 237 Patients With Stage III Non-Small-Cell Lung Cancer. International Journal of Radiation Oncology, Biology, Physics, 73, 1383-1390. http://dx.doi.org/10.1016/j.ijrobp.2008.06.1935

[20] Wurstbauer, K., Deutschmann, H., Dagn, K., et al. (2013) DART-Bid (Dose-Differentiated Accelerated Radiation Therapy, 1.8 Gy Twice Daily)—A Novel Approach for Non-Resected NSCLC: Final Results of a Prospective Study, Correlating Radiation Dose to Tumor Volume. Radiation Oncology, 8, 49. http://dx.doi.org/10.1186/1748-717X-8-49 